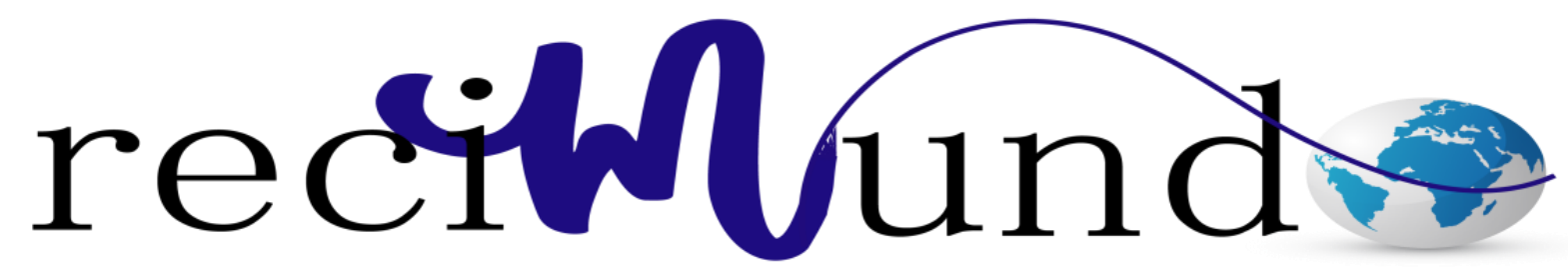

Revista Científica Mundo de la Investigación y el Conocimiento

César Enrique Arreaga Pérez ${ }^{\text {a }}$, Gabriela Del Cisne Valdivieso Samaniego ${ }^{\text {b; Milton }}$

Fernando Cobos Zambrano $^{c}$; Génesis Belén Tumbaco Guerrero ${ }^{\mathrm{d}}$

Síndrome de apnea del sueño en pediatría

Pediatric sleep apnea syndrome

Revista Científica Mundo de la Investigación y el Conocimiento. Vol. 3 núm.3, septiembre, ISSN: 2588-073X, 2019, pp. 201-223

DOI: 10.26820/recimundo/3.(3).septiembre.2019.201-223

URL: $\underline{\text { http://recimundo.com/index.php/es/article/view/518 }}$

Código UNESCO: 3205 Medicina Interna

Tipo de Investigación: Artículo de Revisión

Editorial Saberes del Conocimiento

Recibido: 15/05/2019

Aceptado: 23/06/2019

Publicado: 30/09/2019

Correspondencia: cesarreaga.88@gmail.com

a. Médico; Investigador Independiente; Guayaquil, Ecuador; cesarreaga.88@ gmail.com

b. Especialista en Pediatría; Médica General; Investigador Independiente; Guayaquil, Ecuador; gabrielavaldiviesos@gmail.com

c. Médico; Investigador Independiente; Guayaquil, Ecuador; dr.fernandocobos@gmail.com

d. Médico; Investigador Independiente; Guayaquil, Ecuador; dra.genesistumbaco@gmail.com 


\section{Síndrome de apnea del sueño en pediatría}

Vol. 3, núm. 3., (2019)

César Enrique Arreaga Pérez; Gabriela Del Cisne Valdivieso Samaniego; Milton Fernando Cobos Zambrano; Génesis Belén Tumbaco Guerrero

\section{RESUMEN}

La prevalencia del síndrome de apnea del sueño en niños tiene mayor incidencia en preescolares. Se caracteriza por una obstrucción parcial o completa de la vía aérea superior durante el sueño, que causa hipoxia intermitente. Ambos factores anatómicos (obstrucción nasal severa, anomalías craneofaciales, hipertrofia del tejido linfoide faríngeo, anomalías laríngeas, etc.) y factores funcionales (enfermedades neuromusculares) predisponen al síndrome de apnea del sueño durante la infancia, la principal causa de síndrome de apnea del sueño en niños es la hipertrofia amigdalar. Las manifestaciones clínicas más comunes del síndrome de apnea del sueño son: ronquidos nocturnos, pausas respiratorias, sueño inquieto y respiración bucal. La oximetría de pulso nocturna, grabación de audio / video de ruido nocturno y la polisomnografía de la siesta son herramientas útiles para la detección en casos sospechosos de síndrome de apnea del sueño en niños, y el estándar de oro para el diagnóstico es la polisomnografía nocturna en el laboratorio del sueño. Al contrario de los adultos con síndrome de apnea del sueño, los niños suelen presentar: menos excitaciones asociadas a eventos de apnea, apneas / hipopneas más numerosas durante el sueño REM y desaturación de oxihemoglobina más significativa incluso en apneas cortas. El tratamiento del síndrome de apnea del sueño puede ser quirúrgico (adenotonsilectomía, corrección de anomalías craneofaciales, traqueotomía) o clínico (higiene del sueño, presión positiva continua en las vías aéreas CPAP).

Palabras Claves: Apnea; niños; apnea del sueño; sueño; obstrucción de vías aéreas. 
Síndrome de apnea del sueño en pediatría

Vol. 3, núm. 3., (2019)

César Enrique Arreaga Pérez; Gabriela Del Cisne Valdivieso Samaniego; Milton Fernando

Cobos Zambrano; Génesis Belén Tumbaco Guerrero

\begin{abstract}
The prevalence of sleep apnea syndrome in children has a higher incidence in preschoolers. It is characterized by a partial or complete obstruction of the upper airway during sleep, which causes intermittent hypoxia. Both anatomical factors (severe nasal obstruction, craniofacial abnormalities, pharyngeal lymphoid tissue hypertrophy, laryngeal abnormalities, etc.) and functional factors (neuromuscular diseases) predispose to sleep apnea syndrome during childhood, the main cause of sleep apnea syndrome In children it is tonsillar hypertrophy. The most common clinical manifestations of sleep apnea syndrome are: night snoring, respiratory pauses, restless sleep and mouth breathing. Night pulse oximetry, night noise audio / video recording and nap polysomnography are useful tools for detection in suspected cases of sleep apnea syndrome in children, and the gold standard for diagnosis is night polysomnography In the sleep lab. In contrast to adults with sleep apnea syndrome, children usually present: fewer excitations associated with apnea events, more frequent apnea / hypopnea during REM sleep and more significant oxyhemoglobin desaturation even in short apneas. The treatment of sleep apnea syndrome can be surgical (adenotonsilectomy, correction of craniofacial anomalies, tracheotomy) or clinical (sleep hygiene, continuous positive pressure in the CPAP airways).
\end{abstract}

Key Words: Apnea; children; sleep apnea; sleep; airway obstruction. 


\section{Síndrome de apnea del sueño en pediatría}

Vol. 3, núm. 3., (2019)

César Enrique Arreaga Pérez; Gabriela Del Cisne Valdivieso Samaniego; Milton Fernando Cobos Zambrano; Génesis Belén Tumbaco Guerrero

\section{Introducción.}

Varios niños pueden presentar alteraciones respiratorias durante el sueño: ronquido primario, síndrome de resistencia de las vías respiratorias superiores y la apnea hipopneas (apnea central, obstructiva o mixta).

El ronquido primario se caracteriza por el fuerte sonido en las vías respiratorias superiores con marco en el sueño, ventilación de alvéolos y con saturación de oxígeno de la hemoglobina en los niveles normales. Es común en la infancia y afecta del 7 al 9\% de los niños de 1 a 10 años.

El síndrome de resistencia superior de las vías respiratorias se caracteriza por: ronquidos en la noche, despertares frecuentes, sueño fragmentado y aumento de la resistencia a la inhalación del flujo de aire pero sin reducción del flujo significativo o desaturación de la oxihemoglobina. La prevalencia en los niños sigue siendo desconocida. (Bowe \& Buckmiller, 2011)

La apnea central es la interrupción del comando del SNC (Sistema Nervioso Central) a los músculos respiratorios que causa el flujo de aire para dejar en las fosas nasales y la boca la ausencia del esfuerzo respiratorio y los movimientos abdominales o torácicos. Tiene mayor prevalencia en niños recién nacidos y prematuros. Se piensa que no afecta a la saturación de oxígeno en sangre (SpO2) (Marcus, 2007).

La apnea obstructiva es el cese del flujo de aire en las fosas nasales y la boca debido al colapso de las vías respiratorias superiores, sin importar el esfuerzo de los músculos abdominales en el pecho. En niños sanos es un evento raro y durante se duermen se presenta con mayor frecuencia, el proceso no dura más de 10 segundos. La apnea mixta implica la disminución del 


\section{Síndrome de apnea del sueño en pediatría}

Vol. 3, núm. 3., (2019)

César Enrique Arreaga Pérez; Gabriela Del Cisne Valdivieso Samaniego; Milton Fernando

Cobos Zambrano; Génesis Belén Tumbaco Guerrero

control respiratorio central y la obstrucción superior de las vías respiratorias. La hipoapnea es la reducción parcial del flujo de aire en las fosas nasales y la boca y puede ser clasificado también la central, obstructiva o mixta. (Marcus, 2007)

El síndrome de apnea obstructiva del sueño (SAOS) fue descrita por primera vez en los niños por William Osler en 1892, pero su estudio sistematizado ee convirtió sólo después de la década de los 70. De acuerdo con la Clasificación Internacional de Trastornos del Sueño, el SAOS es un "desorden intrínseco del sueño caracterizado por frecuentes episodios de obstrucción de las vías respiratorias superiores inducidos por dessaturation hemoglobina. (Brunetti \& Rana, 2012)

La prevalencia del SAOS en niños varía de $0,7 \%$ a $3 \%$ en diferentes estudios epidemiológicos. El pico de incidencia se encontró en niños en edad preescolar dentro de los actos en los que la hipertrofia de las amígdalas y adenoides son más comunes. El SAOS podría tener graves consecuencias como: cardiopatía pulmonar, índice corporal de bajo de peso, problemas de conducta, bajo rendimiento de aprendizaje en la escuela, y las funciones neurocognitivas pueden verse afectadas. (Gozal, 2015)

\section{Métodos y Materiales.}

Para el desarrollo de este proceso investigativo, se plantea como metodología la encaminada hacia una orientación científica particular que se encuentra determinada por la necesidad de indagar en forma precisa y coherente una situación, en tal sentido (Davila, 2015) define la metodología "como aquellos pasos previos que son seleccionados por el investigador para lograr resultados favorables que le ayuden a plantear nuevas ideas".(p.66) 


\section{Síndrome de apnea del sueño en pediatría}

Vol. 3, núm. 3., (2019)

César Enrique Arreaga Pérez; Gabriela Del Cisne Valdivieso Samaniego; Milton Fernando Cobos Zambrano; Génesis Belén Tumbaco Guerrero

Lo citado por el autor, lleva a entender que el desarrollo de la acción investigativa busca simplemente coordinar acciones enmarcadas en una revisión bibliográfica con el fin de complementar ideas previas relacionadas al Síndrome de apnea del sueño en pediatría, a través de una revisión de literatura, para así finalmente elaborar un cuerpo de consideraciones generales que ayuden a ampliar el interés propuesto.

\section{Tipo de Investigación.}

Dentro de toda práctica investigativa, se precisan acciones de carácter metodológico mediante las cuales, se logra conocer y proyectar los eventos posibles que la determinan, así como las características que hacen del acto científico un proceso interactivo ajustado a una realidad posible de ser interpretada. En este sentido, se puede decir, que la presente investigación corresponde al tipo documental, definido por Castro (2016), "se ocupa del estudio de problemas planteados a nivel teórico, la información requerida para abordarlos se encuentra básicamente en materiales impresos, audiovisuales y /o electrónicos”. (p.41).

En consideración a esta definición, la orientación metodológica permitió la oportunidad de cumplir con una serie de actividades inherentes a la revisión y lectura de diversos documentos donde se encontraron ideas explicitas relacionadas con los tópicos encargados de identificar a cada característica insertada en el estudio. Por lo tanto, se realizaron continuas interpretaciones con el claro propósito de revisar aquellas apreciaciones o investigaciones propuestas por diferentes investigadores relacionadas con el tema de interés, para luego dar la respectiva argumentación a los planteamientos, en función a las necesidades encontradas en la indagación. 


\section{Síndrome de apnea del sueño en pediatría}

Vol. 3, núm. 3., (2019)

César Enrique Arreaga Pérez; Gabriela Del Cisne Valdivieso Samaniego; Milton Fernando

Cobos Zambrano; Génesis Belén Tumbaco Guerrero

\section{Fuentes Documentales.}

El análisis correspondiente a las características que predomina en el tema seleccionado, llevan a incluir diferentes fuentes documentales encargadas de darle el respectivo apoyo y en ese sentido cumplir con la valoración de los hechos a fin de generar nuevos criterios que sirven de referencia a otros procesos investigativos. Para (CASTRO, 2016) las fuentes documentales incorporadas en la investigación documental o bibliográfica, "representa la suma de materiales sistemáticos que son revisados en forma rigurosa y profunda para llegar a un análisis del fenómeno".(p.41). Por lo tanto, se procedió a cumplir con la realización de una lectura previa determinada para encontrar aquellos aspectos estrechamente vinculados con el tema, con el fin de explicar mediante un desarrollo las respectivas apreciaciones generales de importancia.

\section{Técnicas para la Recolección de la Información.}

La conducción de la investigación para ser realizada en función a las particularidades que determinan a los estudios documentales, tiene como fin el desarrollo de un conjunto de acciones encargadas de llevar a la selección de técnicas estrechamente vinculadas con las características del estudio. En tal sentido, (Bolívar, 2015), refiere, que es "una técnica particular para aportar ayuda a los procedimientos de selección de las ideas primarias y secundarias”. (p. 71).

Por ello, se procedió a la utilización del subrayado, resúmenes, fichaje, como parte básica para la revisión y selección de los documentos que presentan el contenido teórico. Es decir, que mediante la aplicación de estas técnicas se pudo llegar a recoger informaciones en cuanto a la revisión bibliográfica de los diversos elementos encargados de orientar el proceso de investigación. Tal como lo expresa, (Bolívar, 2015) "las técnicas documentales proporcionan las herramientas 


\section{Síndrome de apnea del sueño en pediatría}

Vol. 3, núm. 3., (2019)

César Enrique Arreaga Pérez; Gabriela Del Cisne Valdivieso Samaniego; Milton Fernando Cobos Zambrano; Génesis Belén Tumbaco Guerrero

esenciales y determinantes para responder a los objetivos formulados y llegar a resultados efectivos" (p. 58). Es decir, para responder con eficiencia a las necesidades investigativas, se introdujeron como técnica de recolección el método inductivo, que hizo posible llevar a cabo una valoración de los hechos de forma particular para llegar a la explicación desde una visión general.

Asimismo, se emplearon las técnicas de análisis de información para la realización de la investigación que fue ejecutada bajo la dinámica de aplicar diversos elementos encargados de determinar el camino a recorrer por el estudio, según, (Bolívar, 2015) las técnicas de procesamiento de datos en los estudios documentales "son las encargadas de ofrecer al investigador la visión o pasos que debe cumplir durante su ejercicio, cada una de ellas debe estar en correspondencia con el nivel a emplear" (p. 123). Esto indica, que para llevar a cabo el procesamiento de los datos obtenidos una vez aplicado las técnicas seleccionadas, tales como: fichas de resumen, textual, registros descriptivos entre otros, los mismos se deben ajustar al nivel que ha sido seleccionado.

OSAS: obstrucción del sueño por síndrome de apnea

\section{Resultados.}

Las vías respiratorias son permeables, en condiciones fisiológicas, debido a factores anatómicos y funcionales. Cualquier anomalía en tales factores puede desencadenar en la obstrucción del sueño por el síndrome de apnea.

\section{Factores anatómicos.}




\section{Síndrome de apnea del sueño en pediatría}

Vol. 3, núm. 3., (2019)

César Enrique Arreaga Pérez; Gabriela Del Cisne Valdivieso Samaniego; Milton Fernando

Cobos Zambrano; Génesis Belén Tumbaco Guerrero

1. El autor (Finkelstein, Wexler, \& Berger, 2013) en su estudio expresa que las anormalidades óseas craneofacial son el andamiaje que protege las vías respiratorias superiores. Por lo tanto, cualquier malformación tales como atresia de coanas, micrognatca, hipoplasia mandibular y otras anomalías base del cráneo (por ejemplo platibasia) puede causar obstrucción respiratoria.

La micrognacia está relacionada con mas de 60 síndromes genéticos (microsomía hemifacial, Treacher-Collins, Goldenhar, Pierre Robin, etc.) y que con frecuencia coexiste con otras malformaciones y microglosias resultantes en el desplazamiento dorsal de la lengua y el estrechamiento orofaringe.

En los síndromes de anomalías craneofaciales (abajo, Crouzon, síndrome de Apert, Pfeiffer, etc.) la deformidad base del cráneo y la hipoplasia pueden causar obstrucción maxilar de las cavidades nasales y rinofaringe.

Las anormalidades óseas de límites rinofaringe pueden disminuir su diámetro anteroposterior. Posiblemente es la causa de que no todos los niños con trastornos respiratorios tienen hipertrofia de adenoides durante el sueño, sin embargo, algunos otros niños que roncan continúa incluso después de la adenoidectomía.

Anormal posicionamiento dorsal-caudal del hueso hioides en algunos niños es la causa de trastorno respiratorio durante el sueño. Estos pacientes tienden a dormir en posición de hiperextensión cervical en la que se eleva el hioides con el fin de proporcionar un alivio temporal de la obstrucción. 


\section{Síndrome de apnea del sueño en pediatría}

Vol. 3, núm. 3., (2019)

César Enrique Arreaga Pérez; Gabriela Del Cisne Valdivieso Samaniego; Milton Fernando Cobos Zambrano; Génesis Belén Tumbaco Guerrero

2. Anormalidades de tejidos blandos obstrucción nasal grave debido a la rinitis, tumores o pólipos nasales voluminosos puede causar respiración por la boca y obstrucción del sueño por síndrome de apnea (Salib, Sadek, Dutt, \& Pearman, 2010). En el primer año de vida, la cavidad craneal de la laringe y la glotis pueden alcanzar paladar blando. Esta configuración ofrece protección contra el aumento de la aspiración de alimentos si el niño hace movimientos de chupar, sin embargo facilita la obstrucción de la faringe. Los niños con laringomalacia y otras enfermedades de la laringe (webs, tumores) también pueden tener apnea obstructiva.

La anormalidad anatómica más frecuente en los niños con obstrucción del sueño por síndrome de apnea es faríngea hipertrofia del tejido linfático, esto ocurre principalmente entre 3 y 8 años de edad. Cabe señalar que esto parece ser el factor necesario de anormalidad, pero no es suficiente para desencadenar la obstrucción del sueño por síndrome de apnea, ya que, no todos los niños con adenoides y amígdalas tienen apnea hipertrofia. (Marcus, 2011)

La mayoría de los niños con hipertrofia de amígdalas palatinas no presentan obstrucción respiratoria mientras están despiertos, cuando se aumenta el tono muscular, sin embargo, muchos de estos niños son sometidos a adenoamigdalectomía donde los síntomas de obstrucción se presentan de nuevo en la adolescencia. Estos hechos llevan a creer que la obstrucción del sueño por síndrome de apnea puede resultar de la combinación de anormalidades anatómicas y funcionales en ciertos niños. (Marcus C. , 2007)

\section{Factores funcionales.}

Hipotonía de los intercostales y la dilatación de los músculos de las vías respiratorias superiores durante el sueño. En el sueño REM activar el reflejo de los músculos geniogloso y 


\section{Síndrome de apnea del sueño en pediatría}

Vol. 3, núm. 3., (2019)

César Enrique Arreaga Pérez; Gabriela Del Cisne Valdivieso Samaniego; Milton Fernando

Cobos Zambrano; Génesis Belén Tumbaco Guerrero

tensor de paladar blando se reduce o se vuelve ausente resultando una disminución superior del calibre de las vías respiratorias, lo que aumenta su resistencia al flujo aéreo. (Marcus, 2011)

El autor (Harvey, 2002) en su estudio expresa que los factores relacionados con la obstrucción del sueño por síndrome de apnea son probables en pre y peri natal en 40 niños sin trastornos neurológicos, el autor también afirma que cuando aumenta la incidencia de infecciones y complicaciones en el embarazo las madres de niños con obstrucción del sueño por síndrome de apnea necesitan ser ingresadas en hospitales en comparación con las madres de niños sanos. Los niños con obstrucción del sueño por síndrome de apnea también tendían a sufrir más complicaciones perinatales como la hipoxemia y enfermedades respiratorias. El autor también analizó la hipótesis de complicaciones de maternidad y eventos perinatales que da disminución del control neural de la vía aérea superior, determinando que esto puede ser una predisposición a la obstrucción del sueño por síndrome de apnea.

Los niños con enfermedades neuromusculares causadas por hipotonía (distrofia muscular) o la falta de coordinación (parálisis cerebral) generalmente muestran un aumento del riesgo a presentar obstrucción del sueño por síndrome de apnea graves. (Marcus, 2011)

Síntomas clínicos de la obstrucción del sueño por síndrome de apnea.

\section{Sistema cardiovascular}

En un estudio realizado por (Greene \& Carroll, 2008) a 41 niños con obstrucción del sueño por síndrome de apnea se encontró que 32\% tenían sistólica y diastólica (presión arterial) con percentil de 95, tanto en el sueño como cuando estaban despiertos. La hipertensión se relaciona 


\section{Síndrome de apnea del sueño en pediatría}

Vol. 3, núm. 3., (2019)

César Enrique Arreaga Pérez; Gabriela Del Cisne Valdivieso Samaniego; Milton Fernando Cobos Zambrano; Génesis Belén Tumbaco Guerrero

directamente con la gravedad de la apnea obstructiva y el nivel de obesidad en los niños. Los autores atribuyeron el aumento sub-cortical de la presión arterial de estos niños al despertar, y no a la hipoxemia, ya que, no había ninguna relación encontrada entre las mediciones de presión y oximetría. Los autores también señalaron la necesidad de evaluar si el tratamiento de obstrucción del sueño por síndrome de apnea normalizaría a los niños.

La obstrucción de las vías aéreas superiores y la hipoventilación alveolar crónica dan como resultado una relación anormal de ventilación y perfusión pulmonar. La hipercapnia y la hipoxemia causan acidosis respiratoria y posterior vasoconstricción de la arteria pulmonar aumentando la carga de trabajo del ventrículo derecho. Al mismo tiempo, las arterias pulmonares de calibre pequeñas y medias presentan remodelación e hipertrofia de las capas musculares lisas que con el tiempo pueden evolucionar a hipertrofia de miocardio y, en algunos casos, a dilatación del ventrículo derecho, insuficiencia cardíaca y corpulmonale. (Görür \& Döven, 2001)

\section{Crecimiento y metabolismo.}

Existen tres teorías para explicar la aparición de un índice de bajo peso en muchos niños con obstrucción del sueño por síndrome de apnea: 1. disminución de la producción de hormona del crecimiento, 2. disminución de la ingesta calórica debido a la anorexia / disfagia en niños con hipertrofia de amígdalas adenoides, y 3. aumento del gasto energético debido al esfuerzo respiratorio nocturno. (Nieminen, 2002)

La hormona del crecimiento se secreta durante las etapas profundas del sueño REM. Supuestamente, la secreción nocturna de la hormona de crecimiento disminuye en pacientes con obstrucción del sueño por síndrome de apnea. En realidad, el nivel de crecimiento de insulina 1 en 


\section{Síndrome de apnea del sueño en pediatría}

Vol. 3, núm. 3., (2019)

César Enrique Arreaga Pérez; Gabriela Del Cisne Valdivieso Samaniego; Milton Fernando

Cobos Zambrano; Génesis Belén Tumbaco Guerrero

sangre es el factor principal mediador del efecto de la hormona de crecimiento y la proteína de unión al factor de crecimiento de insulina, siendo más bajo en niños con obstrucción del sueño por síndrome de apnea que en niños sanos. Sin embargo, después de someterse a una adenoamigdalectomía, estos niveles se vuelven normales. (Nieminen, 2002)

La autora (Eva, 2012) cree que la descarga simpática causada por episodios de apnea leve produce un aumento de las concentraciones séricas de catecolamina, cortisol e insulina. Al estudiar el metabolismo de 62 niños y adolescentes obesos con obstrucción del sueño por síndrome de apnea, descubrieron que la glucemia rápida era anormal en el 11\% de los casos, directamente relacionada con el índice de apnea e hipoapnea registrado en la polisomnografía.

\section{Funciones cognitivas.}

El aprendizaje y de comportamiento la somnolencia diurna excesiva es una de las principales quejas de pacientes adultos con obstrucción del sueño por síndrome de apnea, ya que, el sueño está fragmentado. Esta queja es menos común en niños con obstrucción del sueño por síndrome de apnea debido al número reducido de despertares y la preservación relativa de la arquitectura del sueño.

La prueba de latencias múltiples del sueño o como sus siglas en ingles (MSLT) es uno de los mejores métodos para evaluar la somnolencia diurna. La latencia promedio del sueño inferior a 10 minutos es un indicador de somnolencia excesiva. El estudio MSLT realizado en 54 niños con obstrucción del sueño por síndrome de apnea (diagnosticados con un índice de apnea> 2) presentó a 14 niños con ronquidos primarios y 24 pacientes con control de salud con latencia del 


\section{Síndrome de apnea del sueño en pediatría}

Vol. 3, núm. 3., (2019)

César Enrique Arreaga Pérez; Gabriela Del Cisne Valdivieso Samaniego; Milton Fernando Cobos Zambrano; Génesis Belén Tumbaco Guerrero

sueño más baja en la primera prueba, sin embargo, solo el 13\% del total de niños con obstrucción del sueño por síndrome de apnea presentaron latencia inferior a 10 minutos. (Gozal, 2015)

Las pruebas de inteligencia, memoria y atención realizadas en 16 escolares referidos al tratamiento de ronquidos presentaron un rendimiento cognitivo pobre en comparación con 16 pacientes sanos de control de la misma edad. Los niños con síntomas de ronquido presentaron déficit de inteligencia y atención incluso sin somnolencia diurna. Se supone que el déficit de atención afecta el procesamiento y el registro de la información, disminuyendo la capacidad de aprendizaje en niños con obstrucción del sueño por síndrome de apnea.

Una encuesta evaluó la prevalencia de trastornos respiratorios mientras dormía en 782 niños con bajo rendimiento en el primer grado de las escuelas públicas primarias de los EE. UU. Los padres respondieron un cuestionario sobre los síntomas respiratorios de sus hijos; posteriormente, los niños se sometieron a oximetría de pulso y capnografía durante la noche. La prevalencia del ronquido primario en esta muestra fue del 22,2\% y los trastornos respiratorios del sueño del 18,1\%. (Blunden, Lushington, \& Kennedy, 2009)

Diagnóstico de la obstrucción del sueño por síndrome de apnea.

1. Historia del paciente: Los principales con síntomas de obstrucción del sueño por síndrome de apnea en niños incluyen ronquidos nocturnos, pausas respiratorias, respiración dificultosa, sueño inquieto, sudores nocturnos y respiración bucal, rinitis, enuresis y dormir en el cuello uterino. 


\section{Síndrome de apnea del sueño en pediatría}

Vol. 3, núm. 3., (2019)

César Enrique Arreaga Pérez; Gabriela Del Cisne Valdivieso Samaniego; Milton Fernando

Cobos Zambrano; Génesis Belén Tumbaco Guerrero

2. Examen físico: Solo la porción superior de las amígdalas del paladar es visible en la orofaringoscopia y puede proporcionar una falsa impresión de su tamaño y forma. Los autores sugieren una radiografía de perfil de las vías aéreas superiores para una evaluación más precisa de la columna de aire y la obstrucción de la amígdala palatina. (Li \& Wong, 2007)

La nasofibroscopia es útil en el examen de la cavidad nasal y la rinofaringe, ya que permite al otorrinolaringólogo diagnosticar la desviación del tabique nasal y la hipertrofia de las conchas nasales y las adenoides, así como los cambios en la dinámica respiratoria y la deglución debido a la hipertrofia de las amígdalas palatinas.

3. Polisomnografía: La polisomnografía (PSG) realizada en el laboratorio del sueño durante toda la noche es el método de diagnóstico estándar más eficaz para determinar la obstrucción del sueño por síndrome de apnea. El examen tiene una excelente repetibilidad, proporciona evidencia de obstrucción de las vías aéreas superiores y diferencia la apnea obstructiva de la apnea central, y registra episodios epilépticos en niños con trastornos neurológicos. Las recomendaciones de la American Thoracic Society para realizar PSG en niños son las siguientes:

Diagnóstico diferencial entre el ronquido primario y el síndrome de apnea obstructiva del sueño.

Evaluación del niño con patrones de sueño patológicos (somnolencia diurna excesiva, p. Ej.). 


\section{Síndrome de apnea del sueño en pediatría}

Vol. 3, núm. 3., (2019)

César Enrique Arreaga Pérez; Gabriela Del Cisne Valdivieso Samaniego; Milton Fernando Cobos Zambrano; Génesis Belén Tumbaco Guerrero

Confirmación diagnóstica de obstrucción respiratoria durante el sueño para recomendación de tratamiento quirúrgico.

Evaluación preoperatoria de los riesgos de complicaciones respiratorias de adenotonsilectomía u otras cirugías de las vías aéreas superiores.

Evaluación de laringomalacia en pacientes, ya que, los síntomas son más intensos durante la noche o en presencia de cor pulmonale.

Evaluación de niños obesos con somnolencia diurna excesiva, ronquidos, policitemia o cor pulmonale.

Evaluación de niños con anemia falciforme (debido al riesgo de oclusión vascular durante el sueño).

Recurrencia del ronquido en la adenoamigdalectomía postoperatoria.

Tratamiento de control periódico de la presión continua de la vía aérea (CPAP).

Hay muchas diferencias que se encuentran en la polisomnografía durante la evaluación de la obstrucción del sueño por síndrome de apnea en niños y adultos. En el caso de los adulto, el episodio de apnea casi siempre es seguido por un despertar cortical que resulta en un sueño fragmentado, pero solo el $20 \%$ de los niños presentaron despertar cortical. La mayoría de los despertares, apnea obstructiva e hipoapnea en niños ocurren durante el sueño REM. Esta 


\section{Síndrome de apnea del sueño en pediatría}

Vol. 3, núm. 3., (2019)

César Enrique Arreaga Pérez; Gabriela Del Cisne Valdivieso Samaniego; Milton Fernando

Cobos Zambrano; Génesis Belén Tumbaco Guerrero

característica es diferente en adultos en los que la obstrucción de las vías aéreas superiores es más común en el sueño no REM. (Marcus, 2011)

Se considera apnea del adulto si los episodios de interrupción del flujo de aire duran 10 segundos o más. En este intervalo de tiempo, un adulto presenta solo dos o tres ciclos respiratorios, sin embargo, los niños pequeños pueden presentar hasta seis ciclos debido a su mayor frecuencia respiratoria. (Marcus C. , 2007)

Los niños sufren desaturación de hemoglobina significativa incluso en apnea a corto plazo; Tienen un mayor metabolismo y consumo de oxígeno que los adultos. Los adultos presentan obstrucción total y cíclica de las vías respiratorias superiores, mientras que los niños, especialmente los que tienen menos de tres años, tienden a tener una obstrucción parcial de larga duración, conocida como hipoventilación obstructiva. (Marcus, 2011)

Ante tales diferencias, los parámetros de análisis de PSG en adultos son inadecuados para los niños. La American Thoracic Society recomienda los siguientes criterios:

- Índice de apnea (AI): número de episodios de apnea obstructiva y mixta con un intervalo mínimo de dos ciclos respiratorios. Expresado en episodios / por hora (considerando el cálculo del tiempo total de sueño). OSAS se diagnostica en niños si IA $>1$ / hora.

- Hipoapnea obstructiva: reducción del cincuenta por ciento $(50 \%)$ del flujo de aire o más asociada con oxihemoglobina desaturación $>4 \%$, o $\mathrm{SaO}<90 \%$ y / o despertar. 


\section{Síndrome de apnea del sueño en pediatría}

Vol. 3, núm. 3., (2019)

César Enrique Arreaga Pérez; Gabriela Del Cisne Valdivieso Samaniego; Milton Fernando Cobos Zambrano; Génesis Belén Tumbaco Guerrero

- Índice de apnea-hipopnea (IAH): la suma del número de apnea obstructiva y mixta e hipoapnea expresado en episodios por hora (considerando el cálculo del tiempo total de sueño). El diagnóstico anormal para niños sería IAH> 1 / hora. El AIA también se conoce como índice de trastorno respiratorio (IDR). (Avelino \& Pereira, 2012)

Los estudios de (Harvey, 2002) clasificaron el SAOS en niños como leve (1> IAH <5 / hora), moderado (5> IAH $<9$ / hora e IAH grave $>10$ / hora.

Los niños con sueño respiratorio, pero con IAH <1, ausencia de desaturación de hemoglobina o hipercapnia durante la PSG se diagnostican como ronquidos primarios.

Tratamiento para tratar la obstrucción del sueño por síndrome de apnea.

1. Tratamiento quirúrgico: La adenoamigdalectomía permite la cura de la obstrucción del sueño por síndrome de apnea en un $75-100 \%$ de los niños con hipertrofia adenotonsilar. (Schechter, 2013). Sin embargo, el riesgo de complicaciones respiratorias en la adenoamigdalectomía postoperatoria es mayor en caso de que la cirugía se recomiende para la la obstrucción del sueño por síndrome de apnea que en la faringoamigdalitis recurrente. Los niños de alto riesgo en términos de complicaciones respiratorias son: lactantes, lactantes con anomalías craneofaciales, síndrome de Down y otros trastornos genéticos, parálisis cerebral, enfermedades neuromusculares, trastornos metabólicos y de acumulación, enfermedad pulmonar obstructiva crónica, corpulmonale y anemia de células falciformes, niños con O Sa < 70\%2 o IAH> 10 / hora. La Asociación Americana de Pediatría recomienda en tales casos que los niños permanezcan en el hospital la noche después de la cirugía para tener un monitoreo continuo de la oximetría de pulso. (Pediatria, 2010) 


\section{Síndrome de apnea del sueño en pediatría}

Vol. 3, núm. 3., (2019)

César Enrique Arreaga Pérez; Gabriela Del Cisne Valdivieso Samaniego; Milton Fernando

Cobos Zambrano; Génesis Belén Tumbaco Guerrero

Aunque la traqueotomía es efectiva para tratar este síndrome, el procedimiento puede tener algunas complicaciones (estenosis del estoma, obstrucción de la cánula por tapones de secreción o formación de tejido de granulación) y aumenta la necesidad de atención familiar para el paciente. Por lo tanto, se recomienda un tratamiento específico para tratar los puntos de obstrucción en la vía aérea superior (cirugías otorrinolaringológicas, ortognáticas y maxilofaciales). (James \& Lian, 2017)

En un estudio realizado por (James \& Lian, 2017) donde se encuestó a 25 niños con cirugía para tratar la micrognatia determinó que tuvieron éxito en el alivio de la obstrucción respiratoria en 23 de los casos, no obstante se requirió la reoperación en 2 de los casos, 8\% de los niños pudieron cerrar la traqueotomía después de la operación.

La uvulectomía y la uvulopalatofaringoplastia pueden realizarse conjuntamente con adenotonsillectomía para tratar la obstrucción del sueño por síndrome de apnea, según la evaluación de otorrinolaringología y los miembros del equipo multidisciplinario.

Estos procedimientos son aplicables en enfermedades neuromusculares y en parálisis cerebral.

2. Tratamiento clínico: Se recomienda la presión positiva continua en las vías respiratorias (CPAP nasal o BiPAP) confirmado por la Asociación Americana de Pediatría (Pediatria, 2010) siempre que:

- Exista ausencia de hipertrofia adenotonsilar,

- El tratamiento de apnea quirúrgica no está indicado; 


\section{Síndrome de apnea del sueño en pediatría}

Vol. 3, núm. 3., (2019)

César Enrique Arreaga Pérez; Gabriela Del Cisne Valdivieso Samaniego; Milton Fernando Cobos Zambrano; Génesis Belén Tumbaco Guerrero

- La obstrucción del sueño por síndrome de apnea es persistente después del tratamiento quirúrgico.

Se recomienda una nueva prueba de PSG de seis a ocho semanas después de la operación para confirmar la persistencia de obstrucción del sueño por síndrome de apnea. El CPAP también se puede usar temporalmente en la obstrucción del sueño por síndrome de apnea graves asociados con síndromes genéticos, mucopolisacaridosis y parálisis cerebral hasta que el niño esté listo para la cirugía. (Marcus, 2011)

El CPAP nasal no ha sido aprobado por la "Administración de Drogas y Alimentos" para ser utilizado en niños que pesen menos de $30 \mathrm{~kg}$. Las pautas para la higiene del sueño, el tratamiento de la obesidad y la rinitis también son importantes en el enfoque de tratamiento de los niños con el síndrome. Los efectos crónicos de la respiración bucal deben corregirse con un esfuerzo multidisciplinario conjunto con terapia del habla y / u ortodoncia para restablecer los patrones normales de respiración y el crecimiento craneofacial. (Motonaga, Berti, \& AnselmoLima, 2014)

\section{Conclusiones.}

Los estudios sobre los trastornos respiratorios del sueño en los niños se han desarrollado cada vez más en las últimas décadas. Sin embargo, existen algunos desequilibrios de diagnóstico y tratamiento de las enfermedades y en la práctica clínica.

El tiempo de identificación para el diagnostico de la obstrucción del sueño por síndrome de apnea es mayor en países subdesarrollados. Debido al la brecha entre la aparición de los 


\section{Síndrome de apnea del sueño en pediatría}

Vol. 3, núm. 3., (2019)

César Enrique Arreaga Pérez; Gabriela Del Cisne Valdivieso Samaniego; Milton Fernando

Cobos Zambrano; Génesis Belén Tumbaco Guerrero

síntomas y el diagnostico el cual podría llegar a los tres años de edad del infante aumentando el riesgo de complicaciones cardiovasculares y metabólicas, así como el deterioro de las funciones cognitivas y de aprendizaje escolar. Mientras tanto, la calidad de vida general de la familia empeora debido a la angustia respiratoria y la incomodidad del niño durante el sueño.

Durante el desarrollo de la investigación se observó que la prueba de diagnóstico estándar más eficaz para este síndrome es la polisomnografía nocturna (PSG) en un laboratorio de sueño. La "American Thoracic Society" recomienda que se realice una PSG siempre que se requiera confirmación diagnóstica para evaluar el riesgo quirúrgico de los niños que se someten a una adenoamigdalectomía y para controlar el tratamiento clínico y quirúrgico de la obstrucción del sueño por síndrome de apnea. Sin embargo, el número de centros de atención calificados para realizar PSG no es muy elevado, lo que resulta en la necesario llevar a los pacientes a grandes centros en los que la lista de espera de los pacientes es larga. Sin dejar de mencionar que los costo de PSG en las clínicas privadas del sueño son bastante elevados. Por lo tanto, aunque idealmente se debe confirmar el diagnóstico de la obstrucción del sueño por síndrome de apnea en todos los casos sospechosos, en la práctica, la mayoría de los niños que no tienen síntomas de salud adicionales podrían someterse a una adenoamigdalectomía con base en la evaluación del pediatra y el otorrinolaringólogo y en los hallazgos de la radiografía o nasofibroscopia.

Si hay otras enfermedades asociadas (malformación craneofacial, enfermedades cardíacas, neurológicas, etc.), se deben hacer todos los esfuerzos posibles para realizar PSG en el laboratorio del sueño para evaluar la gravedad de la apnea y el riesgo de complicaciones respiratorias en la adenoamigdalectomía postoperatoria. Si la prueba de diagnóstico no está realmente disponible, el 


\section{Síndrome de apnea del sueño en pediatría}

Vol. 3, núm. 3., (2019)

César Enrique Arreaga Pérez; Gabriela Del Cisne Valdivieso Samaniego; Milton Fernando Cobos Zambrano; Génesis Belén Tumbaco Guerrero

médico podría realizar alternativamente oximetría de pulso nocturno y grabación de audio del ruido respiratorio mientras el niño está durmiendo.

La literatura confirmó que la adenotonsilectomía permite la curación de la obstrucción del sueño por síndrome de apnea en más del $75 \%$ de los niños con hipertrofia adenotonsilar con un impacto positivo en su crecimiento, comportamiento y funcionamiento cognitivo. Varios otros procedimientos quirúrgicos para reparar anomalías de las vías respiratorias superiores también presentan buenos resultados $y$, a veces, es posible extirpar posoperatoriamente la traqueotomía.

Es importante tener en cuenta que el otorrinolaringólogo debe tener especial cuidado al operar a niños con obstrucción del sueño por síndrome de apnea asociado con otras afecciones (neurológicas, cardiovasculares, malformaciones, craneofaciales, etc.), ya que presentan un mayor riesgo de complicaciones respiratorias después de la operación de acuerdo a lo citado durante la investigación.

Los procedimientos quirúrgicos deben llevarse a cabo en entornos hospitalarios con dispositivos adecuados y con unidades de cuidados intensivos intensivos (UCI) y unidades de cuidados semi intensivos para controlar adecuadamente a los niños después de la operación.

A pesar de ser costoso, la terapia nasal CPAP o BiPAP brinda beneficios significativos a los niños con OEA asociados con enfermedades neurológicas o musculares que merecen ser mejor estudiadas en lactantes.

Finalmente se puede concluir, que el equipo multidisciplinario (pediatras, neuropediatras y otorrinolaringólogos) tratante de pacientes con este síndrome debe ser consciente de la 


\section{Síndrome de apnea del sueño en pediatría}

Vol. 3, núm. 3., (2019)

César Enrique Arreaga Pérez; Gabriela Del Cisne Valdivieso Samaniego; Milton Fernando

Cobos Zambrano; Génesis Belén Tumbaco Guerrero

importancia del diagnóstico y tratamiento tempranos de la obstrucción del sueño por síndrome de apnea para prevenir complicaciones y mejorar la calidad de vida del niño.

\section{Bibliografia.}

Greene, M., \& Carroll, J. (2008). Blood pressure in children with obstructive sleep apnea. España: Am J Respir Crit Care Med.

Harvey, J. (2002). Factores etiológicos y desarrollo en sujetos con apnea obstructiva del sueño. Los Angeles: J Paediatr Child Health.

James, D., \& Lian, M. (2017). Reconstrucción mandibular en niños con apnea obstructiva del sueño debido a micrognatia. . Plas Reconst Surg, 131-7.

Li, A., \& Wong, E. (2007). Uso del tamaño de las amígdalas en la evaluación de la apnea obstructiva del sueño.

Marcus. (2011). Sleep-disordered breathing in children. Am J Respir Crit Care Med, 16-30.

Marcus, C. (2007). Fisiopatología de la apnea obstructiva del sueño infantil: conceptos actuales. . Respir Physiol 2000, 143-54.

Motonaga, S., Berti, L., \& Anselmo-Lima, W. (2014). Respiração bucal: causas e alterações no sistema estomatognático. . Rev Bras Otorrinolaringol, 373-8.

Nieminen, P. (2002). Growth and biochemical markers of growth in children with snoring and obstructive sleep apnea. Pediatrics .

Pediatria, A. A. (2010). Guía de práctica clínica: diagnóstico y manejo del síndrome de apnea obstructiva del sueño infantil. .

Salib, R., Sadek, S., Dutt, S., \& Pearman, K. (2010). Pólipo antrocoanal que se presenta con apnea obstructiva del sueño y caquexia. Int J Ped Otorrinolaringol , 163-200.

Schechter, M. (2013). Technical report: diagnosis and management of childhood obstructive sleep apnea. . Pediatrics. 